\title{
MRI for differentiating ovarian endometrioid adenocarcinoma from high-grade serous adenocarcinoma
}

Hai Ming Li ${ }^{1,2}$, Jin Wei Qiang ${ }^{1 *}$, Gan Lin Xia ${ }^{2 *}$, Shu Hui Zhao ${ }^{3}$, Feng Hua Ma' ${ }^{1}$ Song Qi Cai ${ }^{1}$, Feng Feng ${ }^{2}$ and $\mathrm{Ai}$ Yan $\mathrm{Fu}^{2}$

\begin{abstract}
Purpose: To investigate magnetic resonance imaging (MRI) features for differentiating ovarian endometrioid adenocarcinoma (OEC) from high-grade serous adenocarcinoma (HGSC).

Materials and methods: Twenty-three patients with 25 OECs and 93 patients with 139 HGSCs confirmed by surgery and pathology underwent conventional MRI and diffusion-weighted imaging (DWI). The MRI features of the tumors, including laterality, size, shape, configuration, signal intensity, ADC value of solid component, enhancement, ascites, synchronous primary cancer (SPC) of the ovary and endometrium, and clinical stage, were evaluated and compared between two groups.

Results: The following characteristics were significantly more common for OECs than HGSCs: unilateral (91.3\% vs $50.5 \%, P<0.001)$, larger mass ( $80.0 \%$ vs $48.2 \%, P=0.005)$, round or oval shape $(64.0 \%$ vs $17.3 \%$, $P<0.001)$, mainly cystic with mural nodules or papillary projections $(72.0 \%$ vs $18.7 \%, \mathrm{P}<0.001)$, cystic component with homogeneous iso- or hyperintensity on T1WI ( $82.6 \%$ vs $4.3 \%, P<0.001)$, moderate enhancement ( $52.0 \%$ vs $26.6 \%, P=0.011$ ), no or mild ascites (91.3\% vs $57.0 \%, P=0.002)$, and SPC (43.5\% vs 4.3\%, $P<0.001)$. The ADC value of the solid component was higher in OECS $\left(0.979 \pm 0.197 \times 10^{-3} \mathrm{~mm}^{2} / \mathrm{s}\right)$ than in HGSCs $\left(0.820 \pm 0.112 \times 10^{-3} \mathrm{~mm}^{2} / \mathrm{s}\right)(P=0.002)$. When a mainly cystic mass with mural nodules or papillary projections was associated with any one of homogeneously iso- or hyperintense cystic component on TIWI, a relatively higher ADC value and SPC, the sensitivity, specificity, accuracy, and positive and negative predictive values for characterizing OEC were 87.0\%, 93.5\%, 92.2\%, 76.9\%, and $96.7 \%$, respectively.
\end{abstract}

Conclusions: Conventional MRI combining DWI is helpful for differentiating OECs from HGSCs.

Keywords: Ovary, Endometroid carcinoma, High-grade serous carcinoma, Magnetic resonance imaging, Diffusion-weighted imaging

\section{Background}

When an adnexal mass is suspected to be an epithelial ovarian carcinoma by magnetic resonance imaging (MRI), the primary concern of clinicians is that the patient has a high-grade serous adenocarcinoma (HGSC), which is the most frequent epithelial carcinoma and is typically aggressive clinically [1]. However, ovarian

\footnotetext{
* Correspondence: dr.jinweiqiang@163.com; ntxg1008@aliyun.com 'Department of Radiology, Jinshan Hospital, Shanghai Medical College, Fudan University, 1508 Longhang Road, Shanghai 201508, China 2Department of Radiology, Nantong Cancer Hospital, Nantong University, 30 North Tongyang Road, Tongzhou District, Nantong, Jiangsu 226361, China
} Full list of author information is available at the end of the article endometrioid carcinoma (OEC), the second most common type of epithelial carcinoma, accounts for $10 \%$ of all epithelial ovarian malignancies, shares similar morphologic features to HGSC [2]. Previous studies have indicated that there are different risk factors, origins, genetic alterations, biological behaviors, clinicopathological characteristics and chemotherapy sensitivities between OEC and HGSC. Clincally, patients with OECs are more likely to have early stage disease and generally have a favorable prognosis [3-6]. However, few studies have focused on the imaging of OEC [7]. As a result, the imaging features of OEC have not been completely 
identified. Furthermore, conventional MRI and diffusion weighted imaging (DWI) have not been investigated for their ability to differentiate between OEC and HGSC. Preoperative imaging differentiation of OEC from HGSC will be helpful for the management of patient with OEC, because a conservative fertility-sparing surgery can be considered for patients with early-stage OEC who wish to preserve fertility [8]. Therefore, this retrospective study evaluated conventional MRI and DWI for distinguishing OEC from HGSC to improve the preoperative characterization and surgical planning of these two distinctive types of ovarian cancers.

\section{Methods}

\section{Clinical data}

The institutional ethical board (Jinshan Hospital, Shanghai Medical College, Fudan University) approved this retrospective study, and informed consent was waived. Between September, 2010 and October, 2014, we searched for the data of patients with OEC at our hospitals information system and picture achiving and communication system. A total of 25 patients with OEC confirmed by surgery and pathology were found. We excluded 2 patients with metastatic OEC. The remaining 23 patients with 25 OECs were reviewed in this study. The mean age of the patients was $54 \pm 9$ years (range, 32-81 years). As a comparison, 93 patients with 139 HGSCs were served as a control group from 126 surgically and pathologically confirmed cases of HGSC at the same period and database. We excluded 15 patients who received chemotherapy before MR scanning, 8 patients who were performed on a $3.0 \mathrm{~T}$ MR scanner, 6 patients with poor image quality and 4 patients without the administration of intravenous contrast. Their mean age was $55 \pm 9$ years (range, 35-78 years), which was not significantly different from the mean age of patients with OEC ( $\mathrm{P}=0.508)$. The diagnosis of SPC was established according to the pathological criteria proposed by Singh [9]. Twelve patients presented with abdominal pain and swelling; eight patients presented with vaginal bleeding; two patients were asymptomatic and were diagnosed during a routine physical examination; and the last patient presented with an abdominal mass. All patients underwent surgery within 2 weeks after completing the MRI scan.

\section{MRI scanning}

MR imaging was performed using a 1.5-T scanner (Avanto or Espree, Siemens, Erlangen, Germany) with a phased-array abdominal coil. The patients laid in a supine positon and breathed freely during acquisition. The sequences were obtained as follows: axial spin echo (SE) T1-weighted imaging (T1WI) [time of repetition (TR)/time of echo (TE), $340 \mathrm{~ms} / 10 \mathrm{~ms}$ ]; axial turbo SE
T2-weighted imaging (T2WI) with and without fat saturation (TR/TE, $8000 \mathrm{~ms} / 83 \mathrm{~ms}$ and $4000 \mathrm{~ms} / 98 \mathrm{~ms}$, respectively); and sagittal and coronal turbo SE T2WI (TR/TE, $8000 \mathrm{~ms} / 98 \mathrm{~ms}$ ). Axial DWI (19 patients with 20 OECs and 83 patients with 124 HGSCs) was obtained with echo planar imaging (TR/TE, $3200 \mathrm{~ms} / 83 \mathrm{~ms}$ ) and b factors of 0 and $1000 \mathrm{~s} / \mathrm{mm}^{2}$. Contrast-enhanced flash 2D T1WI with fat saturation (TR/TE, $196 \mathrm{~ms} / 2.9 \mathrm{~ms}$ ) was performed in the axial, sagittal and coronal planes immediately after the intravenous administration of Gadopentetate dimeglumine (Magnevist; Bayer Schering, Guangzhou, China) at a dose of $0.2 \mathrm{mmol} / \mathrm{kg}$ of body weight and a rate of $2-3 \mathrm{ml} / \mathrm{s}$. The scanning parameters were as follows: 5-mm slice thickness, $1.2-\mathrm{mm}$ gap, $256-320 \times 256-320$ matrix, $250-296 \mathrm{~mm} \times 250-340 \mathrm{~mm}$ field of view and four excitations. The scanning range was from the inferior pubic symphysis to the renal hilum and extended beyond the dome of the tumor in cases with huge masses.

\section{Image analysis}

The MR images were reviewed independently by two radiologists (H.M.L and J.W.Q) with 7 years and 30 years of experience in abdominal imaging, respectively, and were blinded to the original reports (radiology, surgery and pathology). Any discrepancies were resolved by consensus. The following features of the tumors were evaluated: (a) uni- or bilaterality (both ovaries having similarly sized tumors, which indicates the simultaneous development of primary malignancies), size and shape; (b) mass configuration (mainly cystic, less than one-third solid component; mixed cystic-solid, one- to two-thirds solid component; and solid, more than two-thirds solid component); (c) signal intensity (hypo-, iso-, and hyperintensity, referring to the signal of the outer myometrium in solid components; to the signals of muscle and iliac marrow in cystic components on T1WI and T2WI; and to the signals of small intestine and iliac vessel on DWI and ADC maps, respectively); (d) enhancement (mild, moderate or marked by referencing those of the junctional zone and outer myometrium); (e) amount of ascites (none, mild, moderate, and severe) (f) associated findings (uterine endometrial carcinoma); and (g) apparent diffusion coefficient $(\mathrm{ADC})$ value as measured on ADC maps, a circular region of interest (ROI) of at least $1 \mathrm{~cm}^{2}$ was placed at targeted areas with the possibly lowest ADC values in the solid components of the tumor, by referring to conventional MR imagings and avoiding areas such as haemorrhage, necrosis and major vascular structures. At least three measurements were obtained and averaged.

\section{Statistical analysis}

SPSS 16.0 for Windows (SPSS Inc., Chicago, IL, USA) was used for the statistical analysis. The differences between OECs and HGSCs in laterality, shape, mass configuration, 
signal intensity, enhancement, ascites, SPC, and clinical stage were compared using a Pearson chi-square test. The differences in age, mass size, and ADC values between the two groups were compared using the two independentsample Student $t$-test. A P-value less than 0.05 was considered statistically significant. Receiver operating characteristic (ROC) curve analysis was used to determine a cut-off value for differentiating OECs and HGSCs.

\section{Results and discussion}

The maximum diameters of the OECs ranged from 3.7 to $22.5 \mathrm{~cm}$ (mean $11.1 \pm 4.9 \mathrm{~cm}$ ), and those of the HGSCs ranged from 2.5 to $16.8 \mathrm{~cm}(8.1 \pm 3.4 \mathrm{~cm})(\mathrm{P}=0.005)$. The median size of the tumors was $9.9 \mathrm{~cm}$ in OECs versus
$7.6 \mathrm{~cm}$ in HGSCs. A bilateral mass was found in 2 OEC patients and 46 HGSC patients $(\mathrm{P}<0.001)$. According to the International Federation of Gynecology and Obstetrics (FIGO) staging system, 19 patients with OECs were at stage I (82.6\%), 3 were at stage II (13.0\%), and 1 was at stage III (4.4\%). Pathologically, 14 OECs were grade 1,8 were grade 2, and 1 was grade 3 . For the HGSC group, 6 patients were at stage I (6.4\%), 9 were at stage II (9.7\%), 73 were at stage III (78.5\%), and 5 was at stage IV (5.4\%). There was a statistically significant difference in the number of patients at different stages of disease between the two groups $(\mathrm{P}<0.000)$.

The MRI features of OEC and HGSC are summarized in Table 1. The mass was round or oval in 16 of 25

Table 1 MRI morphological features of OEC versus HGSC

\begin{tabular}{|c|c|c|c|}
\hline Morphological features & OEC $(n=25)$ & HGSC $(n=139)$ & $P$ value \\
\hline Laterality^ & & & 0.000 \\
\hline Bilateral & $2(8.7 \%)$ & $46(49.5 \%)$ & \\
\hline Unilateral & $21(91.3 \%)$ & $47(50.5 \%)$ & \\
\hline Shape & & & 0.000 \\
\hline Round/oval & $16(64.0 \%)$ & $24(17.3 \%)$ & \\
\hline Lobulated & $6(24.0 \%)$ & $26(18.7 \%)$ & \\
\hline Irregular & $3(12.0 \%)$ & $89(64.0 \%)$ & \\
\hline Configuration & & & 0.000 \\
\hline Cystic with mural nodules or papilla & $18(72.0 \%)$ & $26(18.7 \%)$ & \\
\hline Mixed cystic-solid & $5(20.0 \%)$ & $43(31.0 \%)$ & \\
\hline Solid & $2(8.0 \%)$ & $70(50.3 \%)$ & \\
\hline SI of solid component on T1WI & & & 0.343 \\
\hline Iso-/hypointense & $24(96.0 \%)$ & $121(87.1 \%)$ & \\
\hline Mainly iso- with hyperintense & $1(4.0 \%)$ & $18(12.9 \%)$ & \\
\hline SI of solid component on T2WI & & & 0.576 \\
\hline Isointense & $3(12.0 \%)$ & $9(6.5 \%)$ & \\
\hline Heterogeneous hyperintense & $22(88.0 \%)$ & $130(93.5 \%)$ & \\
\hline SI of cystic component on T1WI & & & 0.000 \\
\hline Homogeneous hypointense & $4(17.4 \%)$ & $46(66.7 \%)$ & \\
\hline Homogeneous iso-/hyperintense & $19(82.6 \%)$ & $3(4.3 \%)$ & \\
\hline Mainly hypo- with iso-/hyperintense & 0 & $20(29.0 \%)$ & \\
\hline SI of cystic component on T2WI & & & 0.751 \\
\hline Homogeneous hyperintense & $18(78.3 \%)$ & $58(84.1 \%)$ & \\
\hline Mixed signal* & $5(21.7 \%)$ & $11(15.9 \%)$ & \\
\hline Enhancement & & & 0.011 \\
\hline Moderate & $13(52.0 \%)$ & $37(26.6 \%)$ & \\
\hline Marked & $12(48.0 \%)$ & $102(73.4 \%)$ & \\
\hline Ascites^ & & & 0.002 \\
\hline None or mild & $21(91.3 \%)$ & $53(57.0 \%)$ & \\
\hline Moderate or severe & $2(8.7 \%)$ & $40(43.0 \%)$ & \\
\hline $\mathrm{SPC} \wedge$ & $10(43.5 \%)$ & $4(4.3 \%)$ & 0.000 \\
\hline
\end{tabular}

SI: signal intensity; *Mixed signal means that two or more signals within the tumors. ^ numbers for 23 patients with OEC and 93 patients with HGSC. 
(64.0\%) OECs versus 24 of 139 (17.3\%) HGSCs $(\mathrm{P}<0.001)$. The mass configuration was mainly cystic with mural nodules or papillary projections in 18 of $25(72.0 \%)$ OECs versus 26 of $139(18.7 \%)$ HGSCs $(\mathrm{P}<0.001)$. The signal of the cystic component was homogeneously iso- or hyperintense on TIWI in 19 of 23 (82.6\%) OECs versus 3 of $69(4.3 \%)$ HGSCs $(\mathrm{P}<0.001)$ (Figures 1,2 and 3 ). The solid component showed a moderate enhancement in 13 of 25 (52.0\%) OECs versus 37 of 139 (26.6\%) HGSCs $(\mathrm{P}=0.011)$. No or mild ascites were observed in 21 of 23 (91.3\%) OECs versus 53 of 93 (57.0\%) HGSCs $(P=0.002)$ (Figures 4 and 5). SPC was observed in 10 of 23 (43.5\%) OECs versus 4 of 93 (4.3\%) HGSCs $(\mathrm{P}<0.001)$ (Figure 1). Other features, such as the signal intensity of the solid component on T1WI, T2WI, and the signal intensity of the cystic component on T2WI were not significantly different between the two groups $(\mathrm{P}>0.05)$.

On DWI, the solid component showed hyperintensity in 17 of 20 (85.0\%) OECs and isointensity in the remaining 3 (15.0\%), while for HGSCs, the solid component showed hyperintensity in 111 of 116 (95.7\%) and isointensity in 5 (4.3\%) $(\mathrm{P}=0.173)$. The mean ADC value of the solid component was $(0.979 \pm 0.197) \times 10^{-3} \mathrm{~mm}^{2} / \mathrm{s}$ in 20 of 25 OECs versus $(0.820 \pm 0.112) \times 10^{-3} \mathrm{~mm}^{2} / \mathrm{s}$ in 116 of 139 HGSCs $(\mathrm{P}=0.002)$ (Figures $1,3,4$ and 5$)$. ROC curve analysis yielded an optimal ADC value threshold of $0.971 \times$ $10^{-3} \mathrm{~mm}^{2} / \mathrm{s}$ for differentiating OECs from HGSCs, with a sensitivity of $55.0 \%$, a specificity of $94.0 \%$ and an accuracy of $88.2 \%$.

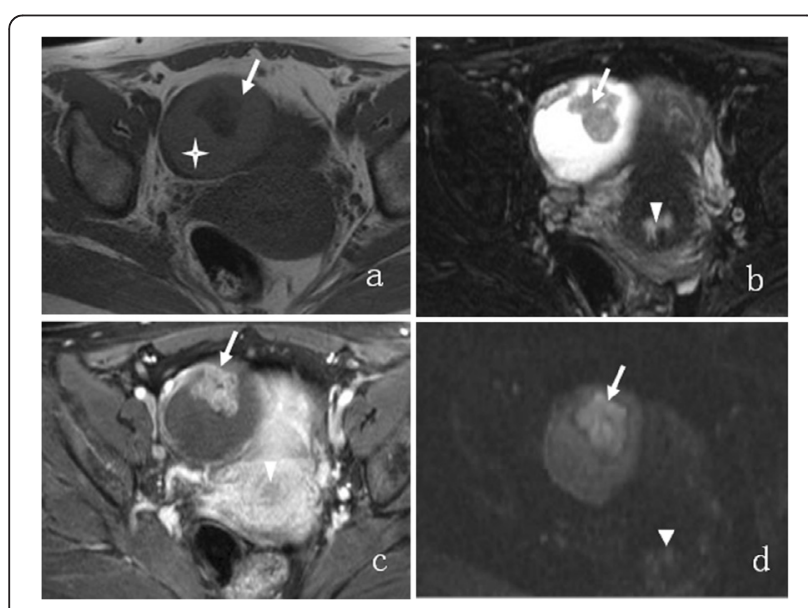

Figure 1 A 50-year-old woman with right-sided OEC. The tumor appears as a mainly cystic mass with a large mural nodule. The cystic component (crossstar) shows slight hyperintensity on axial T1WI (a), homogeneous hyperintensity on axial T2WI with fat suppression (b), no enhancement on contrast-enhanced T1WI with fat suppression (c), isointensity on DWI (d). The mural nodule (arrow) demonstrates isointensity (a), heterogenous hyperintensity (b), marked enhancement (c) and hyperintensity (d) with an ADC value of $1.030 \times 10^{-3} \mathrm{~mm}^{2} / \mathrm{s}$, respectively. Synchronous uterine endometrial carcinoma (arrowhead) shows mild enhancement on contrast-enhanced T1WI and hyperintensity on DWI.
The diagnostic performance of the different MRI features for differentiating OEC from HGSC are listed in Table 2 . When a mainly cystic mass with mural nodules or papillary projections was associated with any one of homogeneously iso- or hyperintense cystic component on TIWI, a higher ADC value, and SPC, the sensitivity, specificity, accuracy, positive and negative predictive values for characterizing OEC were $87.0 \%, 93.5 \%, 92.2 \%, 76.9 \%$, and $96.7 \%$, respectively.

During the past decade, there has been strong interest in type-specific treatment of epithelial ovarian carcinoma, and considerable advances have been achieved in the understanding and differentiation of the five types of ovarian cancers (high-grade serous adenocarcinoma, endometrioid carcinoma, clear cell carcinoma, mucinous carcinoma, low-grade serous adenocarcinoma) [10,11]. Ovarian HGSCs and OECs are two of the most frequent types of epithelial ovarian carcinomas. Studies have indicated that most OECs are diagnosed at an early stage, have a low histologic grade, respond well to chemotherapy, and consequently, have a lower recurrence rate and a better survival compared with HGSCs [5]. Furthermore, studies have suggested that the fertility-preserving surgery has a low currence rate and is safe for the patient with stage I epithelial ovarian carcinoma. There is no difference in the overall survival compared with the radical surgery [12-15]. In contrast, patients with HGSC need radical surgical staging and cytoreduction [8]. Therefore, discriminating OEC from HGSC is essential for preoperative surgical planning, especially in stage I patients who wish to preserve fertility and/or female endocrine functions.

The present MRI study showed that OECs were significantly different from HGSCs in laterality, size, shape, configuration, signal intensity, enhancement, ADC value, SPC, clinical stage and ascites. OEC commonly appeared as a large, unilateral, round or oval cystic mass with mural nodules or papillary projections, homogeneous iso- or hyperintensity on T1WI in the cystic component, and moderate enhancement and relatively higher ADC values in the solid component. OEC was commonly associated with SPC and was diagnosed at an early stage. In contrast, HGSC was typically a moderately sized, irregular solid or mixed cystic-solid mass with marked enhancement and a lower ADC value, more common bilaterality and moderate to severe ascites. Although some features had low diagnostic specificity, four features, a cystic mass with mural nodules or papillary projections, homogeneously iso- or hyperintense cystic component on T1WI, higher ADC value and SPC, have a high specificity for OEC. Those four features yielded a sensitivity, specificity, accuracy, positive and negative predictive values for characterizing OEC of $87.0 \%, 93.5 \%, 92.2 \%, 76.9 \%$, and $96.7 \%$, respectively. 

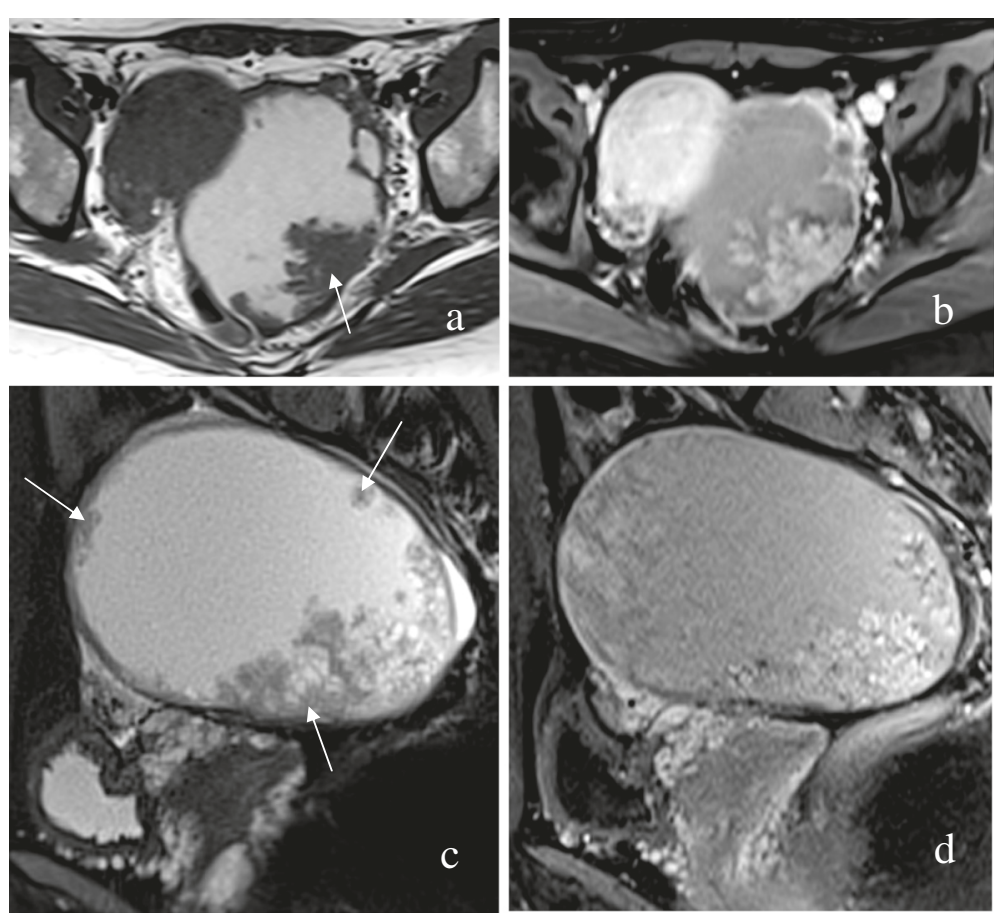

Figure 2 A 32-year-old woman with left-sided OEC. Axial and sagittal T2WI (a, c) demonstrate a mainly cystic mass with multiple mural nodules (arrows). Axial and sagittal contrast-enhanced T1WI with fat suppression $\mathbf{( b ,} \mathbf{d})$ show that the nodules are moderately enhanced.

Histopathologically, it has been well-established that atypical endometriosis is a precursor lesion for OEC. OEC associated endometriosis is found in $20 \%-40 \%$ of cases [16]. In contrast, most ovarian HGSCs are confirmed to be derived from the tubal intraepithelial lining [17], and only $7 \%$ of cases have a history of ovarian endometriosis [18]. In our study, only $13.0 \%(3 / 23)$ of OECs were shown histopathologically to have arisen from an endometriotic cyst, an incidence lower than that of a previous study (33.3\%) [7]. A possible explanation for this result is insufficient sampling due to the study not being pathogenesis-oriented [19].

A previous study showed that $33.3 \%$ of OECs were cystic, and the remaining $66.7 \%$ were mainly solid, which was inconsistent with our findings [7]. Selection bias and misdiagnosis are the possible causes because research has found that 50 of 176 (28\%) formerly diagnosed OECs are actually HGSCs [20]. The cystic component displayed homogeneous iso- or hyperintensity on T1WI in $82.6 \%$ $(19 / 23)$ of OECs versus $4.3 \%$ of HGSCs and was another important differentiating feature. The iso- or hyperintensity on T1WI signifies bloody cystic content, which may imply OEC is derived from endometriosis [21].

In our study, almost all OECs and HGSCs demonstrated high signal in the solid component on DWI. The mean ADC value of the solid component was $(0.979 \pm 0.197) \times$ $10^{-3} \mathrm{~mm}^{2} / \mathrm{s}$ in OECs versus $(0.820 \pm 0.112) \times 10^{-3} \mathrm{~mm}^{2} / \mathrm{s}$ in HGSCs, and this difference was statistically significant. An optimal ADC value threshold of $0.971 \times 10^{-3} \mathrm{~mm}^{2} / \mathrm{s}$ yielded a sensitivity, specificity and accuracy of $55.0 \%$,

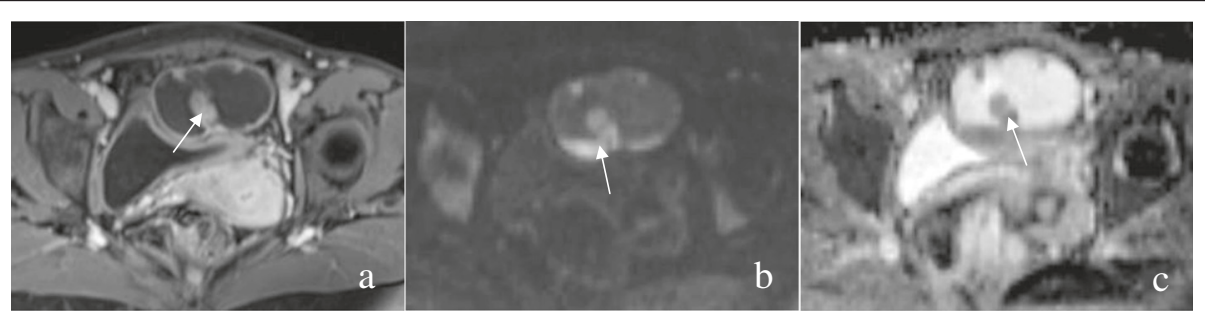

Figure 3 A 53-year-old woman with left-sided OEC. Contrast-enhanced T1WI with fat suppression (a) demonstrates a mainly cystic mass with mural nodules (arrow), which show a marked enhancement (a) and a hyperintensity on DWI (b) with an ADC value of $1.174 \times 10^{-3} \mathrm{~mm}^{2} / \mathrm{s}(\mathbf{c})$. 


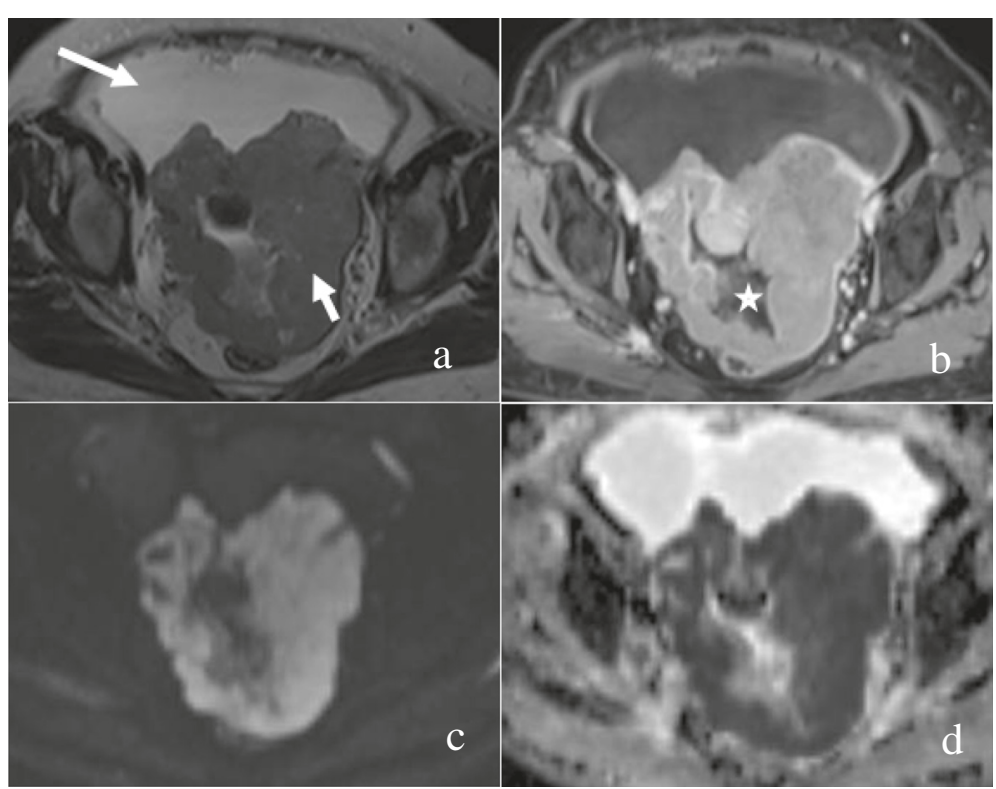

Figure 4 A 67-year-old woman with left-sided HGSC. Axial T2WI (a) demonstrates a solid mass (short arrow) with an irregular shape and a large volume of ascites (long arrow). The mass is markedly enhanced with irregular areas of necrosis (asterisk) on contrast-enhanced T1WI with fat suppression (b), hyperintensity on DWI (c) and hypointensity on ADC map with an ADC value of $0.682 \times 10^{-3} \mathrm{~mm}^{2} / \mathrm{s}$ (d).

94.0\% and $88.2 \%$, respectively, for differentiating OECs from HGSCs, In our study, the solid component in OECs was mainly mural nodules or papillary projections, which were reported to have relatively low malignant potential [22]. Histopathologically, the mural nodules or papillary projections had a loose structure with fewer tumor cells, a fibrous axis, and interstitial edema; this explains the higher ADC value of OECs [23]. In contrast, the solid component in HGSCs was mainly a solid mass that histopathologically contained substantial tumor cells resulting in restricted movement of water molecules and, consequently, a lower ADC value.

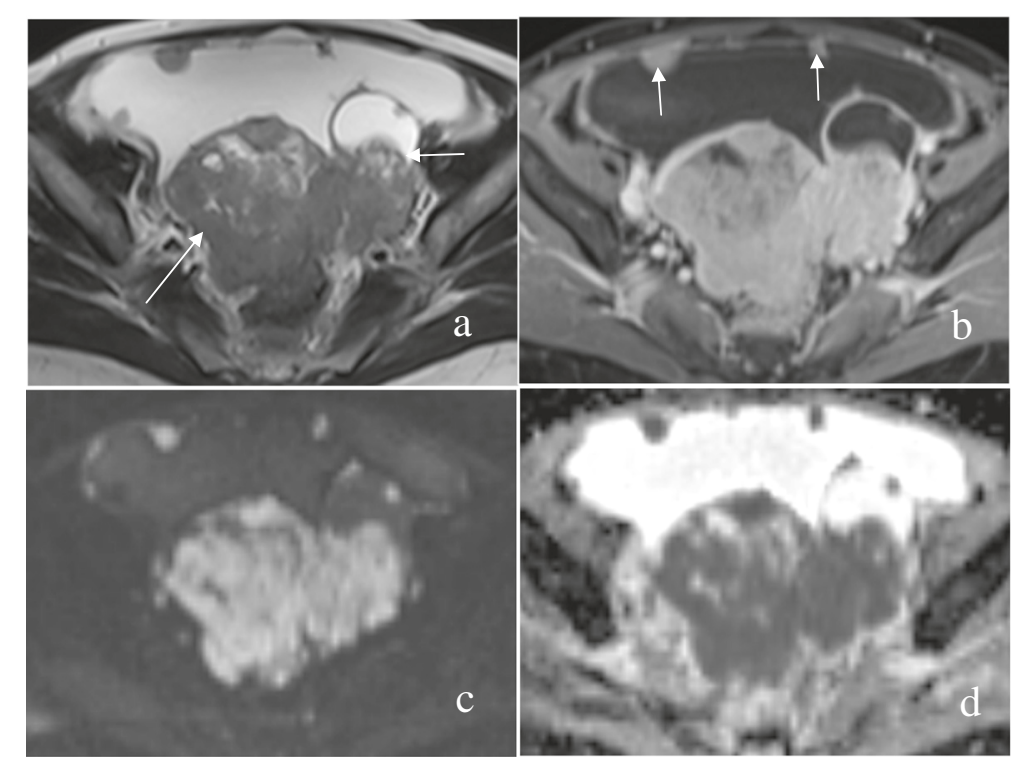

Figure 5 A 57-year-old woman with bilateral HGSC. Axial T2WI (a) demonstrates a solid mass (long arrow) in the right-sided ovary and a mixed cystic-solid mass (short arrow) in the left-sided ovary, which have an irregular shape. A large volume of ascites is observed. The solid components of the masses and peritoneal nodules (arrows) are enhanced markedly on contrast-enhanced T1WI with fat suppression (b), hyperintensity on DWI (c) and hypointensity on ADC map (d) with an ADC value of $0.759 \times 10^{-3} \mathrm{~mm}^{2} / \mathrm{s}$ (left) and $0.782 \times 10^{-3} \mathrm{~mm}^{2} / \mathrm{s}$ (right). 
Table 2 Diagnostic performance of MRI for differentiating OEC from HGSC

\begin{tabular}{llllll}
\hline MRI features & Sensitivity & Specificity & Accuracy & PPV(\%) & NPV(\%) \\
\hline Mass size $(\geq 7.8 \mathrm{~cm})$ & $80.0(20 / 25)$ & $51.8(72 / 139)$ & $56.1(92 / 164)$ & $23.0(20 / 87)$ & $93.5(72 / 77)$ \\
Unilateral & $91.3(21 / 23)$ & $49.5(46 / 93)$ & $57.8(67 / 116)$ & $30.9(21 / 68)$ & $95.8(46 / 48)$ \\
Round/Oval shape & $64.0(16 / 25)$ & $82.7(115 / 139)$ & $79.9(131 / 164)$ & $40.0(16 / 40)$ & $92.7(115 / 124)$ \\
Mainly cystic mass & $72.0(18 / 25)$ & $81.3(113 / 139)$ & $79.9(131 / 164)$ & $40.9(18 / 44)$ & $94.2(113 / 120)$ \\
Homogeneous iso-/hyperintensity* & $82.6(19 / 23)$ & $95.7(66 / 69)$ & $92.4(85 / 92)$ & $86.4(19 / 22)$ & $94.3(66 / 70)$ \\
Moderate enhancement & $52.0(13 / 25)$ & $73.4(102 / 139)$ & $70.1(115 / 164)$ & $26.0(13 / 50)$ & $89.5(102 / 114)$ \\
Ascites(none or mild) & $91.3(21 / 23)$ & $43.0(40 / 93)$ & $52.6(61 / 116)$ & $28.4(21 / 74)$ & $95.2(40 / 42)$ \\
SPC & $43.5(10 / 23)$ & $95.7(89 / 93)$ & $85.3(99 / 116)$ & $71.4(10 / 14)$ & $87.3(89 / 102)$ \\
ADC value^ & $55.0(11 / 20)$ & $94.0(109 / 116)$ & $88.2(120 / 136)$ & $61.1(11 / 18)$ & $92.4(109 / 118)$ \\
\hline PPV: Postive
\end{tabular}

PPV: positive predictive value; NPV: negative predictive value; *the signal intensity of cystic component on T1Wl; $\wedge$ ADC value of solid component $\left(\geq 0.971 \times 10^{-3} \mathrm{~mm}^{2} / \mathrm{s}\right)$, ADC values of solid components are measured and averaged in 20 OECs and $116 \mathrm{HGSCs}$.

Approximately $1-2 \%$ of gynecological malignant tumor cases have two or more synchronous primary genital tract carcinomas [8]. Approximately $10 \%$ of women with ovarian cancer will be found to have synchronous endometrial cancer, and approximately $5 \%$ of women with endometrial cancer harbor simultaneous ovarian cancer [24]. Synchronous OEC and endometrial cancer accounts for $50-70 \%$ of SPC $[8,25]$. On MR imaging, endometrial cancer of SPC often displayed superficial or no myoinvasion. In the present study, the synchronous primary endometrial carcinomas were found in $43.5 \%$ of OEC patients versus $4.3 \%$ of HGSC patients, which indicated high specificity for OEC. However, other studies found synchronous primary endometrial carcinomas in $16 \%$ of OEC patients [7].

Our study had several limitations. First, only imaging features of a limited number of patients were evaluated. Therefore, larger samples are necessary to confirm the value of these features for diagnosing OEC. Second, due to the retrospective design of our study, a selective bias was inevitable. Third, a correlation analysis of MRI features with pathology was not performed.

\section{Conclusions}

In conclusion, our preliminary study demonstrates that a large, round or oval, mainly cystic mass with moderately enhanced mural nodules or papillary projections, a homogeneously iso- or hyperintense cystic component on TIWI, a relatively higher $\mathrm{ADC}$ value in the solid component and, commonly, SPC are features that help to differentiate OEC from HGSC. Although the diagnostic performance of any feature alone is not sufficient for diagnosis, the combination of the feature of a mainly cystic mass with mural nodules or papillary projections with any one of the following features: homogeneously iso- or hyperintense cystic component on TIWI, a relatively higher
ADC value and SPC, yields high sensitivity, specificity and accuracy for identifying OEC.

\section{Abbreviations}

OEC: Ovarian endometrioid adenocarcinoma; HGSC: High-grade serous adenocarcinoma; SPC: Synchronous primary cancer of the ovary and endometrium.

\section{Competing interests}

The authors declare that they have no competing interests.

\section{Authors' contributions}

Guarantor of integrity of entire study, JWQ; study concepts/study design or data acquisition or data analysis/interpretation, all authors; manuscript drafting or manuscript revision for important intellectual content, HML, JWQ; approval of final version of submitted manuscript, all authors; literature research, HML; clinical studies, all authors; statistical analysis, HML, FF; and manuscript editing, HML. All authors read and approved the final manuscript.

\section{Acknowledgements}

Contract grant sponsor: National Natural Science Foundation of China (Grant No.81471628); Shanghai Municipal Commission of Science \& Technology (Grant No.124119a3300) and Shanghai Municipal Commission of Health and Family Planning (Grant No. 2013SY075, Grant No. ZK2012A16).

\section{Author details}

'Department of Radiology, Jinshan Hospital, Shanghai Medical College, Fudan University, 1508 Longhang Road, Shanghai 201508, China. 2Department of Radiology, Nantong Cancer Hospital, Nantong University, 30 North Tongyang Road, Tongzhou District, Nantong, Jiangsu 226361, China. ${ }^{3}$ Department of Radiology, Xinhua Hospital, Shanghai Medical College, Jiaotong University, 1665 Kongjiang Road, Shanghai 200092, China.

Received: 24 January 2015 Accepted: 23 April 2015

Published online: 30 April 2015

\section{References}

1. Feigenberg T, Clarke B, Virtanen C, Plotkin A, Letarte M, Rosen B, et al. Molecular profiling and clinical outcome of hig-grade serous ovarian cancer presenting with low-grade serous ovarian cancer with low-versus highvolume ascites. Biomed Res Int. 2014;2014:367103. doi:10.1155/2014/367103.

2. Lalwani N, Prasad SR, Vikram R, Shanbhogue AK, Huettner PC, Fasih N. Histologic, molecular and cytogenetic features of ovarian cancers: implications for diagnosis and treatment. Radio Graphics. 2011;31:625-46.

3. Lim D, Oliva E. Precursors and pathogenesis of ovarian carcinoma. Pathology. 2013;45:229-42. 
4. Prat J. Ovarian carcinomas: five distinct diseases with different origins, genetic alterations, and clinicopathological features. Virchows Arch. 2012;460:237-49.

5. Storey DJ, Rush R, Stewart M, Rye T, Al-Nafussi A, Williams AR, et al. Endometrioid epithelial ovarian cancer: 20 years of prospectively collected data from a single center. Cancer. 2008;112:2211-20.

6. Wang S, Qiu L, Lang JH, Shen K, Huang HF, Pan LY, et al. Prognostic analysis of endometrioid epithelial ovarian cancer with or without endometriosis: a 12-year cohort study of Chinese patients. Am J Obstet Gynecol. 2013:209:241. e1-e9.

7. Kitajima K, Kaji Y, Kuwata Y, Imanaka K, Sugihara R, Sugimura K. Magnetic resonance imaging findings of endometrioid adenocarcinoma of the ovary. Radiat Med. 2007;25:346-54.

8. Morgan RJ Jr, Armstrong DK, Alvarez RD, Chen LM, Copeland L, Crispens MA, et al. Ovarian cancer: including Fallopian Tube Cancer and Primary Peritoneal Cancer. Version 1. 2014. National Comprehensive Cancer Network; 2014.

9. Singh N. Synchronous tumours of the female genital tract. Histopathology. 2010;56:277-85.

10. McCluggage WG. Morphological subtypes of ovarian carcinoma: a review with emphasis on new developments and pathogenesis. Pathology. 2011:43:420-32.

11. Tothill RW, Tinker AV, George J, Brown R, Fox SB, Lade S, et al. Novel molecular subtypes of serous and endometrioid ovarian cancer linked to clinical outcome. Clin Cancer Res. 2008;14:5198-208.

12. Wright JD, Shah M, Mathew L, Burke WM, Culhane J, Goldman N, et al. Fertility preservation in young women with epithelial ovarian cancer. Cancer. 2009;115:4118-26.

13. Satoh $T$, Hatae M, Watanabe $Y$, Yaegashi N, Ishiko O, Kodama S, et al. Outcomes of fertility-sparing surgery for stage I epithelial ovarian cancer: a proposal for patient selection. J Clin Oncol. 2010;28:1727-32.

14. Kajiyama H, Shibata K, Mizuno M, Umezu T, Suzuki S, Nawa A, et al. Long-term survival of young women receiving fertility-sparing surgery for ovarian cancer in comparison with those undergoing radical surgery. $\mathrm{Br} J$ Cancer. 2011;105:1288-94.

15. Kajiyama H, Mizuno M, Shibata K, Yamamoto E, Kawai M, Nagasaka T, et al. Recurrence-predicting prognostic factors for patients with early-stage epithelial ovarian cancer undergoing fertility-sparing surgery: a multi-institutional study. Eur J Obstet Gynecol Reprod Biol. 2014;175:97-102

16. Stewart CJ, Walsh MD, Budgeon CA, Crook ML, Buchanan DB. Immunophenotypic analysis of ovarian endometrioid adenocarcinoma: correlation with KRAS mutation and the presence of endometriosis. Pathology. 2013:45:559-66.

17. Zeppernick F, Meinhold-Heerlein I, Shil IM. Precursors of ovarian cancer in the fallopian tube: Serous tubal intraepithelial carcinoma-an update. J Obstet Gynaecol Res. 2014; doi:10.1111/jog.12550.

18. Pearce CL, Templeman C, Rossing MA, Lee A, Near AM, Webb PM, et al. Association between endometriosis and risk of histological subtypes of ovarian cancer: a pooled analysis of case-control studies. Lancet Oncol. 2012;13:385-94.

19. Mansor S, McCluggage WG. Endometrioid adenocarcinoma of the ovary mimicking serous borderline tumor:report of a series of cases. Int J Gynecol Pathol. 2014;33:470-6.

20. Gilks CB, lonescu DN, Kalloger SE, Köbel M, Irving J, Clarke B, et al. Tumor cell type can be reproducibly diagnosed and is of independent prognostic significance in patients with maximally debulked ovarian carcinoma. Hum Pathol. 2008;39:1239-51.

21. Tanaka YO, Okada S, Yaqi T, Satoh T, Oki A, Tsunoda H, et al. MRI of endometriotic cysts in assiciotion with ovarian carcinoma. AJR. 2010;194:355-61.

22. Baert AL, Knauth M, Sartor K. MRI and CT of the Female Pelvis. New York: Springer; 2007
23. Outwater EK, Huang AB, Dunton CJ, Talerman A, Capuzzi DM. Papillary projections in ovarian neoplasms: appearance on MRI. J Magn Reson Imaging. 1997;7:689-95.

24. Soliman PT, Slomovitz BM, Broaddus RR, Sun CC, Oh JC, Eifel PJ, et al. Synchronous primary cancers of the endometrium and ovary: a single institution review of 84 cases. Gynecol Oncol. 2004;94:456-62.

25. Tong SY, Lee YS, Park JS, Bae SN, Lee JM, Namkoong SE, et al. Clinical analysis of synchronous primary neoplasms of the female reproductive tract. Eur J Obstet Gynecol Reprod Biol. 2008;136:78-82.

\section{Submit your next manuscript to BioMed Central and take full advantage of:}

- Convenient online submission

- Thorough peer review

- No space constraints or color figure charges

- Immediate publication on acceptance

- Inclusion in PubMed, CAS, Scopus and Google Scholar

- Research which is freely available for redistribution 